\title{
NUMERICAL MODELING OF THERMOCAPILLARY DEFORMATION AND FILM BREAKDOWN IN A LOCALLY HEATED THIN HORIZONTAL VOLATILE LIQUID LAYER
}

\author{
Ella Barakhovskaya $^{1, *}$, and Igor Marchuk ${ }^{1,2}$ \\ ${ }^{1}$ Novosibirsk State University, 630090 Novosiborsk, Russia \\ ${ }^{2}$ Kutateladze Institute of Thermophysics SB RAS, 630090 Novosibirsk, Russia
}

\begin{abstract}
The problem of thermocapillary deformation and film breakdown in a thin horizontal layer of viscous incompressible liquid with a free surface is considered. The deformable liquid layer is locally heated. The problem of thermocapillary deformation of the locally heated horizontal liquid layer has been solved numerically for two-dimensional unsteady case. The lubrication approximation theory is used. Capillary pressure, viscosity and gravity are taken into account. Evaporating rate is supposed to be proportional to the temperature difference between the liquid and ambient. Heat transfer in the substrate is also simulated. The numerical algorithm for the joint solution of the energy equation and the evolution equation for the thickness of liquid layer has been developed. The model predicts the thermocapillary deformation of the liquid surface and the formation of dry spots. The dynamics of liquid surface, the dry spots formation and the velocity of the contact line have been calculated. The deformation of the free surface has been calculated for different values of the heating power and thickness of the liquid layer. The effect of surface tension coefficient and wetting contact angle on the velocity of the contact line motion has been analyzed. It has been obtained that the velocity of the contact line increases with the increase of the wetting contact angle value and of the surface tension coefficient.
\end{abstract}

\section{Introduction}

Heat transfer and film breakdown in thin liquid layer with local heating is an important challenge of thermal stabilization technique of electronic equipment [1-3]. The study of thermocapillary effects in the thin locally heated liquid layer is demanded problem in the field of technological processes as the thin films provide a high intensity of heat and mass transfer. But thin films are exposed to rupture [2,4]. The problem of thermocapillary breakdown of the locally heated horizontal liquid layer has been solved numerically in axisymmetric statement, fig.1. The thin layer approximation model is used [5]. Capillary pressure, viscosity and gravity are taken into account. Evaporating rate is supposed to be

\footnotetext{
${ }^{*}$ Corresponding author: ella94@,bk.ru
} 
proportional to the temperature difference between temperatures of the liquid surface and ambient. Heat transfer in the substrate is also simulated. Initially the liquid layer has flat surface and uniform temperature. The initial thickness of the liquid layer varies from $100 \mu \mathrm{m}$ to a few millimeters. The local heating of the liquid occurs from the substrate side. Deformations of the liquid surface are determined by the properties of the liquid, substrate and heater.

\section{Problem statement}

The problem considers a thin horizontal volatile liquid layer on the substrate with local heater, fig. 1. Liquid is viscous and incompressible. Heater is thin and uniform. Twodimensional unsteady problem is considered. Geometry and system conditions are axisymmetric. Initially the liquid layer has flat surface and uniform constant temperature $T_{0}$. At the time $t=0$ the heater is activated and starts heating the substrate and liquid. Shear stress occurs on the liquid surface caused by the heterogeneity of its temperature. Thermocapillary flow and deformations of the liquid surface are developing. Gravity, surface tension, thermocapillary effect, viscosity, evaporation have a significant influence on the process.

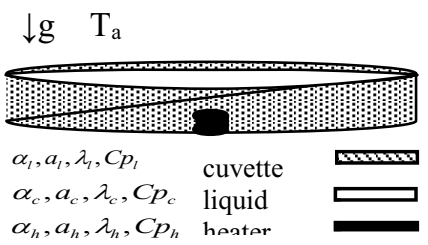

Fig. 1. Scheme of the process.

\section{Mathematical formulation}

\subsection{Motion equation of the liquid}

The mathematical formulation of the problem includes the Navier-Stokes equations, continuity and thermal conductivity. The system of equations in two-dimensional nonstationary case and in cylindrical coordinates [8]:

$$
\left\{\begin{array}{l}
\frac{\partial u}{\partial t}+u \frac{\partial u}{\partial r}+v \frac{\partial u}{\partial z}=F_{r}-\frac{1}{\rho} \frac{\partial p}{\partial r}+v \Delta(r u) \\
\frac{\partial v}{\partial t}+u \frac{\partial v}{\partial r}+v \frac{\partial v}{\partial z}=F_{z}-\frac{1}{\rho} \frac{\partial p}{\partial z}+v \Delta v \\
\frac{1}{r} \frac{\partial(r u)}{\partial r}+\frac{\partial v}{\partial z}=0 \\
\rho C_{p}\left(\frac{\partial T}{\partial t}+u \frac{1}{r} \frac{\partial(r T)}{\partial r}+v \frac{\partial T}{\partial z}\right)=\frac{1}{r} \frac{\partial}{\partial r}\left(\lambda\left(r \frac{\partial T}{\partial r}\right)\right)+\frac{\partial^{2} T}{\partial z^{2}}+Q
\end{array},\right.
$$

The dimensional variables that are used into equations: $u, v-$ the components of liquid velocity vector, $F_{r}=(g \cdot \tau), F_{z}=(g \cdot n)$ - the components of the vector of external forces, $\tau$ - tangent vector along the surface, $\mathrm{n}$ - the normal vector to the surface, $\rho$ - density, $p$ - 
capillary pressure, $v$ - kinematic viscosity, $Q$ - bulk density of the heat sources, $\lambda$ coefficient of thermal conductivity; $C_{p}$ - specific heat, $T$ - temperature, $t$ - time.

\subsection{The evolution equation for liquid film thickness}

Dynamics of thin films is well described by the evolution equation, which has been obtained by the system of equations (1). The evolution equation for liquid film thickness is obtained in the lubrication approximation theory $[5,6]$ :

$$
h_{t}+\operatorname{divq}+J=0
$$

$h_{t}$ - velocity of the surface, $q=\frac{h^{3}}{3 \mu} f+\frac{h^{2}}{2 \mu} \tau$ - liquid flow rate along the $r$ direction, $f=\operatorname{grad}(\rho g h+\sigma H)$ - gradient of the modified pressure, $\tau=\sigma_{T} \operatorname{grad} T$ - thermocapillary tangent stress, $J=J_{e v}^{*}\left(T_{s}(x)-T_{a}\right)$ - evaporation velocity, $T_{s}(x)$ - temperature of the surface, $T_{a}$ - temperature of ambient, $J_{e v}^{*}=\alpha_{e v} /\left(r_{l v} \rho\right)$ - evaporation rate coefficient, $\alpha_{e v}$ evaporation heat transfer coefficient, $r_{l v}$ - latent heat of vaporization, $\mu$ - dynamic viscosity, $\rho$ - density, $\mathrm{g}$ - gravity vector, $\mathrm{h}$ - film thickness, $\sigma$ - surface tension, $\sigma_{\mathrm{T}}$ - linear coefficient, which determines the dependence of the surface tension on temperature, $T$ - temperature, $H=\frac{h_{r r}}{\left(1+h_{r}^{2}\right)^{3 / 2}}+\frac{h_{r}}{r\left(1+h_{r}^{2}\right)^{1 / 2}}-$ double mean curvature.

Substituting the expression for the curvature, liquid flow rate along the surface and evaporation is obtained the equation in cylindrical coordinates:

$$
h_{t}+\frac{1}{r} \frac{\partial}{\partial r}\left(r\left[\frac{h^{3}}{3 \mu} \frac{\partial}{\partial r}(\rho g h+\sigma H)+\frac{h^{2}}{2 \mu} \sigma_{T} \frac{\partial T}{\partial r}\right]\right)+\frac{\alpha_{e v}}{r_{l v} \rho} \Delta T=0
$$

Equation (3) is a nonlinear differential equation of the first order in time and the fourth order in spatial variables relative to unknown function $h(t, r)$.

\subsubsection{The boundary conditions}

Boundary conditions for this problem are set naturally:

$h_{r}(t, 0)=0$ the condition of the axial symmetry is given in the center of the cuvette

$h_{r}(t, R)=0$ the contact angle is given on the border of the cuvette

$q(t, 0)=0$ flow rate is equal to 0 in the center

$q(t, R)=0$ the condition of impermeability of liquid through the walls

Also it is necessary to set boundary conditions in case of occurrence of film breakdown and formation of dry spots:

$$
\begin{aligned}
& h_{r}\left(t, r_{\text {Dry Spot }}\right)= \pm \tan \theta \\
& q\left(t, r_{\text {Dry Spot }}\right)=0
\end{aligned}
$$




\subsection{The energy equation}

The law of temperature distribution in the liquid layer is determined by the energy equation in cylindrical coordinates:

$$
\rho C_{p}\left(\frac{\partial T}{\partial t}+u \frac{1}{r} \frac{\partial\left(r T_{r}\right)}{\partial r}+v \frac{\partial T_{z}}{\partial z}\right)=\frac{1}{r} \frac{\partial}{\partial r}\left(\lambda\left(r \frac{\partial T_{r}}{\partial r}\right)\right)+\frac{\partial^{2} T_{z}}{\partial z^{2}}+Q
$$

$Q=Q(t, r, z)=\left\{\begin{array}{l}Q=0, \text { outside the heater } \\ Q=\text { const }>0, \text { in the heater }\end{array}\right.$ - bulk density of the heat sources, $\lambda$ coefficient of thermal conductivity; $C_{p}$ - specific heat; $\rho$ - density of the liquid.

\subsubsection{The boundary and initial conditions}

The convective heat transfer coefficient is set on the substrate walls:

$$
\left.\lambda \frac{\partial T}{\partial n}\right|_{W}=\alpha\left(T-T_{a}\right)
$$

where $\mathrm{W}$ indicates to conditions on solid surfaces.

In the condition of the top boundary the heat flux density is determined by the evaporation, fig. 2 .

$$
\left.\lambda \frac{\partial T}{\partial z}\right|_{z=h_{w}+h_{l}}=\alpha_{e v}\left(T-T_{a}\right)
$$

$\alpha_{e v}=J_{e v}^{*} r_{l v} \rho$ - the heat transfer coefficient for evaporation, $r_{l v}$ - latent heat of vaporization, $J_{e v}^{*}$ - evaporation rate coefficient. The coefficients for evaporation rates have been calculated using the data published in [7]: $J_{e v}^{*}=3.8 \mu \mathrm{m} / \mathrm{s} \cdot \mathrm{K}, \alpha_{e v}=9.72 \mathrm{~kW} / \mathrm{m}^{2} \mathrm{~K}$.

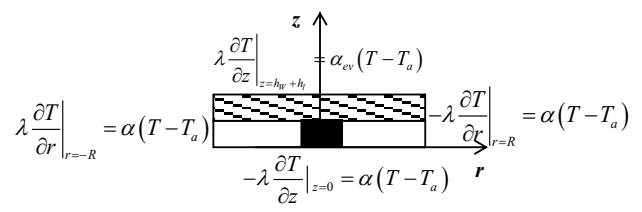

Fig. 2. Boundary conditions.

Initially the liquid layer has flat surface and uniform temperature:

$$
\left.h\right|_{t=0}(r, z)=h_{0},\left.\quad T\right|_{t=0}(r, z)=T_{0}=\text { const }
$$

\section{Numerical modeling}

For calculation it is used splitting into physical processes: distribution of heat and motion of liquid.

The evolution equation of the liquid layer thickness (3) is approximated at grid's nodes with finite volume method [5]. The grid with uniform steps in the space variables in the liquid and solid phases is set: $r_{i}=i \cdot d r, i=0, . ., N$. The time step $\tau$ is also uniform. The value of the liquid layer thickness in the time $t_{k}$ and $i$ node: $h_{i}^{k}=h\left(r_{i}, t_{k}\right)$. A discrete analogue of eq. (3) is written for each volume $V_{i}$ : 


$$
\frac{h_{i}^{k+1}-h_{i}^{k}}{\tau}+\frac{1}{r_{i}} \frac{q_{i+1 / 2} r_{i+1 / 2}-q_{i-1 / 2} r_{i-1 / 2}}{d r}+J_{e v}^{*} \Delta T_{i}=0
$$

System of nonlinear algebraic equations (7) that has been obtained by the approximation is solved with Newton's method. The Jacobians are calculated using numerical linearization [5]. The scheme has the second-order approximation for the spatial coordinates and the first-order in time.

The temperature in the liquid is calculated without allowance of the deformations. Since the heater is thin, it is considered that $Q=Q(t, r, z)$ is concentrated only in heater units. The difference analog of the energy equation (4) is solved by the method of fractional steps [9]:

$$
\left\{\begin{array}{l}
\frac{T_{i j}^{n+1 / 2}-T_{i j}^{n}}{d t}=\frac{\lambda}{2 \rho C p}\left(\frac{\left(1+\frac{d r}{2 r}\right) T_{i+1, j}^{n+1 / 2}-2 T_{i j}^{n+1 / 2}+\left(1-\frac{d r}{2 r}\right) T_{i-1, j}^{n+1 / 2}}{d r^{2}}+\frac{\left.T_{i, j+1}^{n}-2 T_{i j}^{n}+T_{i, j-1}^{n}\right)}{d z^{2}}\right)+\frac{q_{i j}^{n}}{2 \rho C p} \\
\frac{T_{i j}^{n+1}-T_{i j}^{n+1 / 2}}{d t}=\frac{\lambda}{2 \rho C p}\left(\frac{\left(1+\frac{d r}{2 r}\right) T_{i+1, j}^{n+1 / 2}-2 T_{i j}^{n+1 / 2}+\left(1-\frac{d r}{2 r}\right) T_{i-1, j}^{n+1 / 2}}{d r^{2}}+\frac{T_{i, j+1}^{n+1}-2 T_{i j}^{n+1}+T_{i, j-1}^{n+1}}{d z^{2}}\right)+\frac{q_{i j}^{n}}{2 \rho C p}
\end{array}\right.
$$

Boundary and initial conditions are set in the finite differences equations:

$j=1, . ., M$

$T(j, 0)=T(j, 2),-$ symmetry

$T(j, N+1)=T(j, N-1)-$ adiabatic right wall

$i=1, . ., N$

$T(0, i)=T(2, i)-\frac{\alpha}{\lambda}\left(T(1, i)-T_{a}\right) 2 d h$-heat transfer coefficient is given on the free liquid surface

$T(M+1, i)=T(M-1, i)-\frac{\alpha_{w}}{\lambda_{w}}\left(T(M, i)-T_{a}\right) 2 d h_{w}$-heat transfer coefficient is given on bottom wall

Initial condition: $T_{i, j}^{0}=0, i=0, . ., N, j=1, . ., M-1$.

Geometric parameters and parameters of the liquid, substrate and heater are specified. All this properties have a significant effect on the distribution of heat and evolution of liquid deformation. Calculations were performed for ethanol. The initial temperature of the liquid and ambient temperature is $20{ }^{\circ} \mathrm{C}$. The ambient pressure is 1 Bar. The material of the cuvette is Teflon.

The resulting system of linear algebraic equations is solved at each step with the tridiagonal matrix algorithm. Calculations are performed successively. The time step for the evolution equation is done after the time step for the energy equation.

\section{Numerical results}

The deformation of the free surface has been calculated for different values of the heating power and thickness of the liquid layer. A dry spot is formed in a ring shape because of the axial symmetry, fig. 3. Surrounded by a dry ring liquid in the center is a drop, which tends to acquire an equilibrium shape, which is defined by Young-Laplace equation. Then the drop evaporates completely. The dynamics of the three phase contact line is calculated, 
fig. 3. It was obtained that the velocity of the contact line increases with the increase of the wetting contact angle value and of the surface tension coefficient.

a)

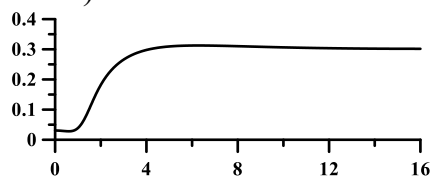

c)

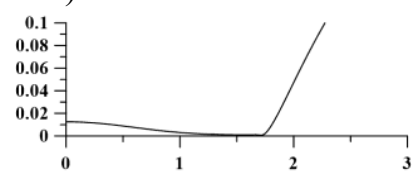

b)

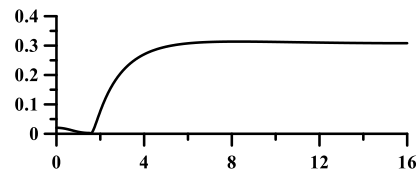

d)

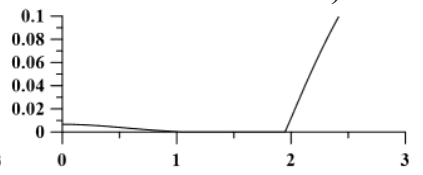

Fig. 3. Liquid layer breakdown and contact line evolution. Ethanol, $h_{0}=300 \mu m$, $D_{\text {cuvette }}=32 \mathrm{~mm}, d_{\text {heater }}=3.2 \mathrm{~mm}, P=0.3 \mathrm{~W}$, heating time: a) $\left.\left.\left.0.6 \mathrm{~s}, \mathrm{~b}\right) 0.9 \mathrm{~s}, \mathrm{c}\right) 1.2 \mathrm{~s}, \mathrm{~d}\right) 1.5 \mathrm{~s}$.

\section{Conclusions}

The numerical calculations with given model of thin horizontal liquid layer with local heating have shown that local heating of a horizontal liquid layer causes deformation of the liquid surface and thermocapillary flow. The model predicts the thermocapillary deformation of the liquid surface and the formation of dry spots with the intensive heating. Significant reduction of liquid film thickness occurs in the greatest value of the temperature gradient zone. Dry the spot is formed in the shape of a ring, but in the center is still liquid, which is surrounded by a dry spot. This liquid takes the form of droplets and ultimately evaporates completely. The velocity of the contact line increases with the increase of the wetting contact angle value and of the surface tension coefficient.

\section{Acknowledgments}

The study was financially supported by the Russian Science Foundation (Project 15-1920049).

\section{References}

1. O.A. Kabov, E.A. Chinnov, High temp. 39, 5 (2001)

2. O.A. Kabov, Yu.V. Lyulin, I.V. Marchuk, D.V. Zaitsev, International J. Heat Fluid Flow 28 (2007)

3. I. V. Marchuk, JET 18, 3 (2009)

4. D.V. Zaitsev, D.A. Rodionov, O.A Kabov, Microgravity sci. technol. 19, 3 (2007)

5. I.V. Marchuk, JET 24, 4 (2015)

6. A. Oron, S.H. Davis, S.G. Bankoff, Rev. Mod. Phys. 69, 3 (1997)

7. A.A. Fedorets, O.A. Kabov, I.V. Marchuk, TPL 37, 2 (2011)

8. L.D. Landau, E.M. Lifshitz, Hydrodynamics, (1988)

9. N.N. Yanenko, The Method of Fractional Steps, (1971) 